\title{
Haematocrit heterogeneity in blood flows past microfluidic models of oxygenating fibre bundles
}

\author{
R. Gómez Bardón ${ }^{\text {a }}$, A. Passos ${ }^{\text {b }}$, M. Piergiovanni ${ }^{\text {a, }}$, S. Balabani ${ }^{\text {b }}$, G. Pennati ${ }^{\text {a }}$, G. Dubini ${ }^{\text {a }}$ \\ a Laboratory of the Biological Structure Mechanics (LaBS), Department of Chemistry, Materials and Chemical Engineering "Giulio Natta", Politecnico di Milano, Milan, Italy \\ ${ }^{\mathrm{b}}$ Department of Mechanical Engineering, University College London (UCL), London, UK
}

\section{A R T I C L E IN F O}

\section{Article history:}

Received 15 February 2019

Received in revised form 11 June 2019

Accepted 19 July 2019

Available online $\mathrm{xxx}$

Keywords:

Red blood cell distribution

Blood oxygenators

Micro fluid dynamics

\begin{abstract}
A B S T R A C T
Blood oxygenators act as an extracorporeal artificial lung during certain types of cardiac surgery and intensive care therapies. Inside these devices, blood is forced to flow across an oxygenating bundle, encountering interstitial gaps comparable to those typical of the microvasculature. Despite the well-known effects of such length scales on haemorheology and red blood cell (RBC) behavior, these are generally overlooked in oxygenator modeling and design; it is persistently assumed that RBCs are homogeneously distributed throughout the oxygenating bundle, independently of their microstructure arrangement or main flow directions.

The goal of this study is to provide preliminary experimental evidence of heterogeneous RBC distributions inside oxygenating fibre bundles. To this end, a number of microchannels were manufactured inspired by actual oxygenating devices, considering simplified versions of their microstructure. These comprise staggered arrays of micro pillars, which were perfused with RBC suspensions, with feed haematocrit (Ht) and velocities relevant for clinical use. The microchannels were imaged using a microscope and high-speed camera to accurately capture cell distribution.

The imaged blood flows revealed the non-uniform nature of RBC distributions in the arrays, characterized by local $\mathrm{Ht}$ gradients particularly around the $\mathrm{O}_{2}$ sources inside the bundle. These heterogeneous distributions should be accounted for during oxygenator design, as RBC concentration plays a key role in $\mathrm{O}_{2}$ transport and, ultimately, overall device performance.
\end{abstract}

\section{Introduction}

The fluid dynamics inside oxygenating fibre bundles is a key element for blood oxygenator performance, typically characterized by highly complex patterns that mainly depend on the microstructure and manufacturing techniques of these devices. Due to the strong coupling between mass transfer mechanisms and blood shear stresses with flow features, an accurate description of local phenomena is crucial in order to predict efficiency and identify possible risk of blood damage.

Typically, modern oxygenating bundles contain hollow fibres made from a semipermeable membrane, which carry gas inside (intraluminal flow), while blood flows externally around them (extraluminal flow). Thanks to the microporous nature of the membrane, gaseous exchange between both phases is possible, so that $\mathrm{O}_{2}$ and $\mathrm{CO}_{2}$ levels in blood remain controlled. This way, an oxygenator can temporarily substitute the functions of the natural lungs, both in cardiac surgery and intensive care therapies. Today most oxygenating bundles are manufactured from a continuous mat of hollow fibres typically arranged in bi-layers of alternating angles, which are either piled or wound around an inner rigid core. The main purpose of such an intricate distribution is to enhance blood mixing, preventing the

\footnotetext{
* Corresponding author.

Email address: monica.piergiovanni@polimi.it (M. Piergiovanni)
}

development of large boundary layers that would limit the mass transfer $[1,2]$.

An additional complication regarding oxygenating bundle analysis is the range of different orders of magnitude and length scales involved. A single adult device can contain around 10,000 fibres [3], with diameters ranging between 200 and $400 \mu \mathrm{m}$. Assuming a representative fibre pitch of $0.6 \mathrm{~mm}$ and a fibre diameter of $0.38 \mathrm{~mm}$ yields a fibre spacing of $220 \mu \mathrm{m}$. However, if the contact between fibres of adjacent layers is considered, the average gaps representative of the blood flow through the bundle are expected to be even smaller. Thus, if different cross sections of a typical wound bundle are studied, irregular patterns with inter-fibre spacing in the order of $50 \mu \mathrm{m}$ can be observed (Fig. 1).

One of the traditional assumptions in computational models describing blood flow in oxygenators is a homogeneous distribution of blood particles throughout the bundle. Although some studies have applied different non-Newtonian shear thinning rheological models (including Ballyk [4,5], Quemada [6] or Casson [7]) to simulate the haemodynamics in different fibre microstructure arrangements, none of these models can account for the effects arising from the particulate nature of blood. A constant concentration of red blood cells (RBCs) is invariably assumed between the fibres across the entire device, failing to consider the blood microstructure within the complex geometry of the oxygenators.

Nevertheless, it is well known that blood, like most particle suspensions, exhibits a complex behavior in microscale flows mainly 
a

b

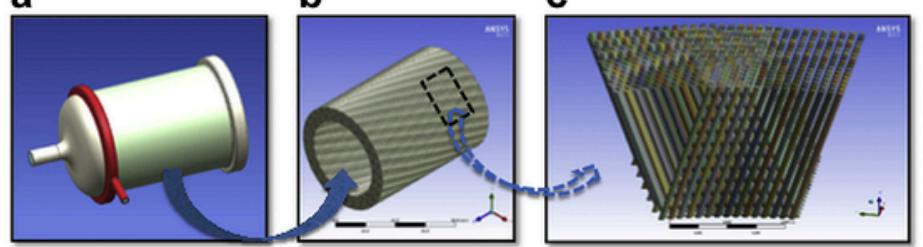

f

d

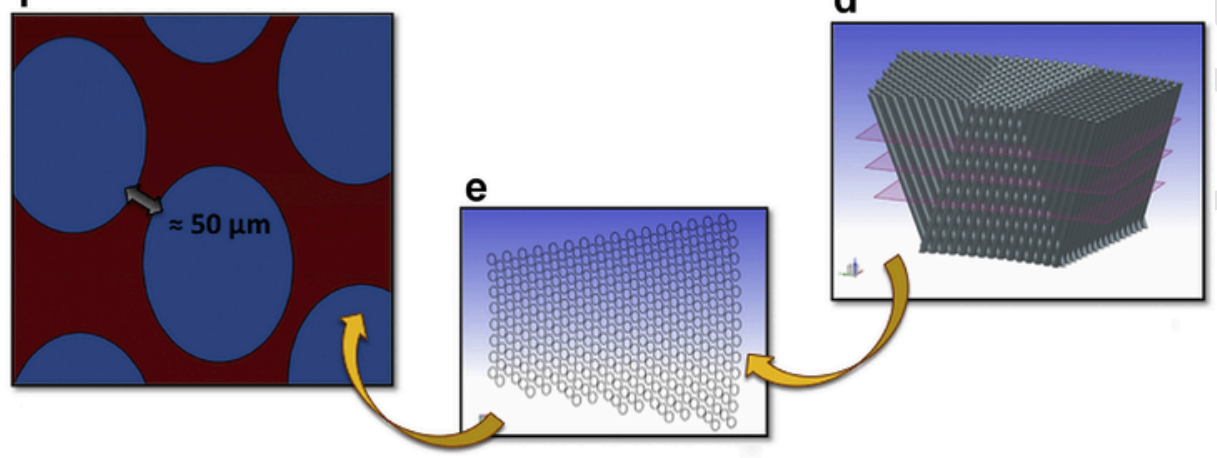

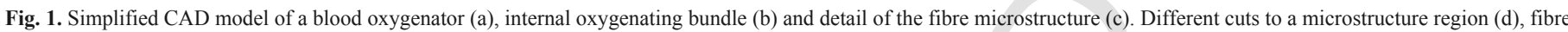

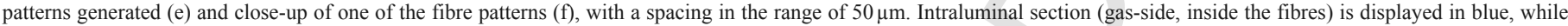

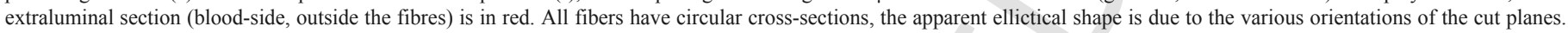
(For interpretation of the references to color in this figure legend, the reader is referred to the web version of this article.)

due to its particulate composition and the properties of the red blood cells [8-10]. It is well established that, due to RBC radial migration, a cell-free layer develops close to the walls giving rise to phenomena such as the Fahraeus effect and a reduction of the apparent viscosity (Fahraeus-Lindqvist) at length scales below $300 \mu \mathrm{m}$ [9,11-13]. These effects are also highly dependent on the properties of RBCs, namely deformability and aggregation, i.e. the propensity of RBCs to form rouleaux at low shear conditions. These phenomena are generally described in the context of microvascular flows and exploited in the context of diagnostics; however, the length scales involved are also representative of extraluminal flow in blood oxygenators (i.e. blood flowing around the oxygenating fibres inside the oxygenator). Although the haematocrit values and Reynolds numbers, are quite different in blood oxygenators $(\mathrm{Re}=1 \div 10)$ compared to microvasculature $(\operatorname{Re}<0.1)$, when considering the typical spacing length scales mentioned above and typical RBC size (diameters of $8 \mu \mathrm{m}$ ), it seems clear that the particulate nature of blood should be of importance, hence compromising the validity of the homogeneous continuum hypothesis for blood description when modeling flows in oxygenating devices.

The present study thus aims to probe the RBC distribution inside oxygenating bundles, with a view to depart from the assumption of a uniform blood composition typically employed in oxygenator design towards more refined modeling approaches for such flows. A number of micropillar array microchannels were designed and manufactured, inspired by the flow paths blood follows inside oxygenators. These might appear as simplified flow configurations; however, a similar approach has been previously adopted in the literature to study $\mathrm{RBC}$ dynamics in biomimetic microfluidic networks of pulmonary alveolar capillaries [21] and spleen [26]. The microchannels were perfused with $\mathrm{RBC}$ suspensions at different haematocrit $(\mathrm{Ht})$ and analyzed through a microscope. In view of the small length scales and high tortuosity of the flow paths, non-uniform RBC distributions are expected to arise which will have major implications in the fluid dynamics therein due to their impact on viscosity $(\mu)$ and density $(\rho)$, as well as in the $\mathrm{O}_{2}$ diffusion through blood across the device. There is a growing interest in the use of multiphase blood models (plasma and
$\mathrm{RBC}$ ) in oxygenator design. A recent computational study suggested a novel and appealing approach for modeling $\mathrm{O}_{2}$ transfer [14], where the RBC and plasma are implemented as two phases. Despite experimental measurements of oxygen transfer in a micro-oxygenator being used to validate the numerical predictions, no direct experimental observation of the blood flow and the local RBC distributions through the fibres was performed. To the best of our knowledge, the results reported below are the first experimental evidence of particulate effects of blood in conditions mimicking those inside oxygenating bundles.

\section{Methods}

\subsection{Geometry definition}

Based on a typical wound bundle microstructure, different microchannel geometries were designed (Fig. 2) as 2D idealizations of the complex patterns shown above. The aim of the experiments was to examine possible gradients of RBC concentration, so simplified geometries were employed as a starting point. The fibres were modeled as micropillar arrays and different features and levels of complexity encountered in oxygenator devices were considered: a straight channel (channel "c1"), a $90^{\circ}$ curved channel ("c2"), and a single row of fibres representing the fibre layer in contact with the oxygenator walls ("c3"). The first model was thought as the simplest case to examine blood flow around the fibre arrays whereas the second one focused on RBC distribution in curved zones, aiming to analyse eventual differences in perfusion of the corner region away from the inlet. Finally, the third simplified geometry aimed to mimic fibres in contact with the walls and hence study the effect of wall boundaries.

Channel $\mathrm{c} 1$ had a total of 367 obstacles (representing circular fibres) distributed in 15 staggered layers, with a total length of $26 \mathrm{~mm}$ and total width of $4.52 \mathrm{~mm}$. Channel c 2 had 776 circular obstacles, in 35 staggered layers, with dimensions $23.5 \times 10 \mathrm{~mm}$. It should be noted that the width of this channel $(10 \mathrm{~mm})$ was chosen according to a realistic dimension of a commercial oxygenator with wound bundles, where the distance between the lateral walls is around $1 \mathrm{~cm}$. Thus, c2 

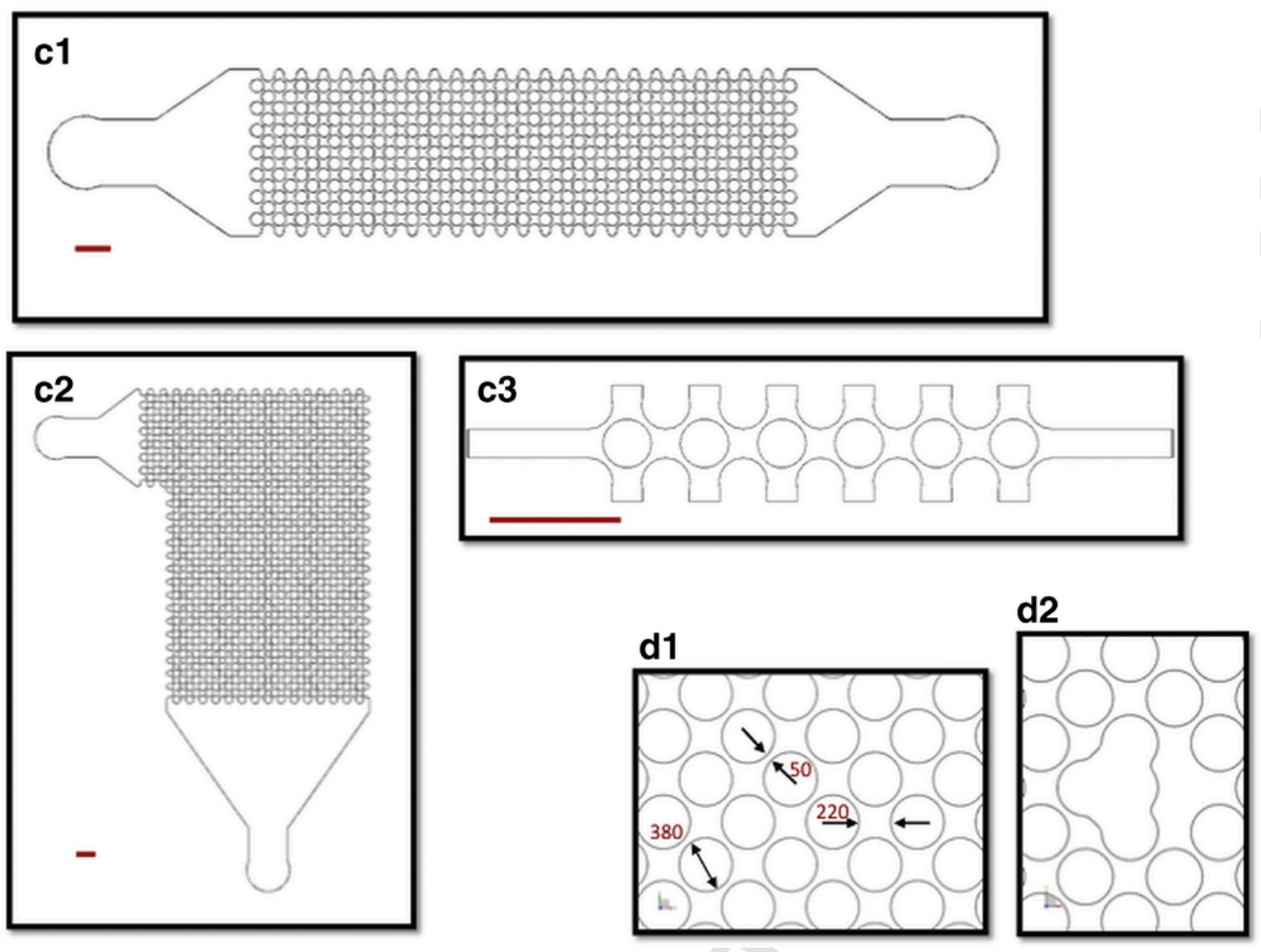

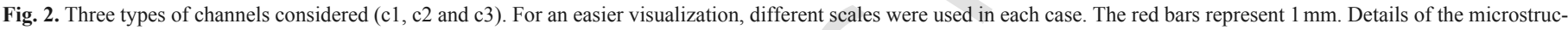

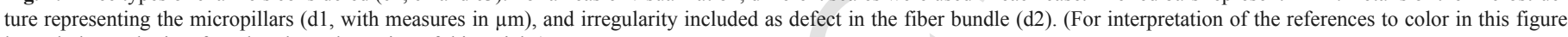
legend, the reader is referred to the web version of this article.)

becomes representative of areas near the inlet/outlet of the device. Finally, c3 is a single micro pin array with 6 obstacles, as a detail of the layer in contact with the wall in $\mathrm{c} 1$.

The diameter of the micropillars was $380 \mu \mathrm{m}$ representative of the fibres in typical oxygenating bundles [3], with maximum and minimum inter-fibre gaps of 220 and $50 \mu \mathrm{m}$, thus resulting in longitudinal and transverse pitch ratios of 0.13 and 0.58 , respectively, with a void fraction of $37 \%$, which is in line with commercial devices. Finally, irregular features representing isolated "defects" in the oxygenating membrane were included. This could correspond to an individual fibre collapsed or misaligned with reference to the other fibres.

For simplicity, the micropillars will be referred to as "fibres" henceforth, even though the only material used in the channels was PDMS, without any real fibre embedded in them.

\subsection{Microfabrication}

Micro-channels were manufactured in a clean room using a standard soft-lithography microfabricating technique for PDMS-based microfluidic devices [15]. The settings were fixed according to a target channel height of $50 \mu \mathrm{m}$ for $\mathrm{c} 1$ and $\mathrm{c} 2$ models, and $40 \mu \mathrm{m}$ for $\mathrm{c} 3$. These values were chosen so that the average aspect ratio of the blood path inside the channels was close to one, as typical of the oxygenating bundles, where the gaps through which the blood flows are not predominantly larger in a particular direction of space.

\subsection{Experimental setup}

Microfluidic channels were placed under an inverted microscope (Nikon Diaphot) and perfused using a flow-controlled syringe pump (Harvard Apparatus PHD ULTRA ${ }^{\mathrm{TM}}$ ). The flow rates used ranged from 70 to $1200 \mu \mathrm{l} / \mathrm{min}$, generating average velocities in the microchannels in the order of $0.1 \mathrm{~m} / \mathrm{s}$ and leading to a Reynolds number always higher than $1\left(\operatorname{Re}=1.7\right.$, assuming $\rho=1025 \mathrm{~kg} / \mathrm{m}^{3}, \mu=0.003$ $\mathrm{Pa} \cdot \mathrm{s}$ and a gap of $50 \mu \mathrm{m}$ between two fibres). This is in line with typical Re inside oxygenating bundles $[7,16,17]$. The flow rates were selected based on the average blood velocity around the fibres, calculated from each channel dimensions and cross-sectional area.

The blood flow through the micropillar arrays was imaged using brightfield illumination and a high-speed CMOS camera (Dantec Dynamics Nano Sense MKIII) with $\times 4, \times 10$ and $\times 20$ objective lenses. The camera sampling rate and exposure time played an important role in the quantification of haematocrit. It should be noted that the aim of this study was to create image intensity maps from which to extract haematocrit information rather than resolve individual RBCs; thus, values of $1000 \mathrm{~Hz}$ and $50 \mu$ s respectively were deemed to be optimal for the conditions of the experiments. A schematic of the experimental set up is shown in Fig. 3.

Preliminary experiments were performed using bovine fresh blood, while human fresh blood was employed in a second set of experiments for a more quantitative analysis. The samples were obtained by venepuncture from healthy donors following an ethics protocol approved by the South East London Research Ethics Committee (Reference: 10/H0804/21). RBCs were separated by centrifugation and suspended in phosphate buffered saline (PBS, Sigma, USA). The 


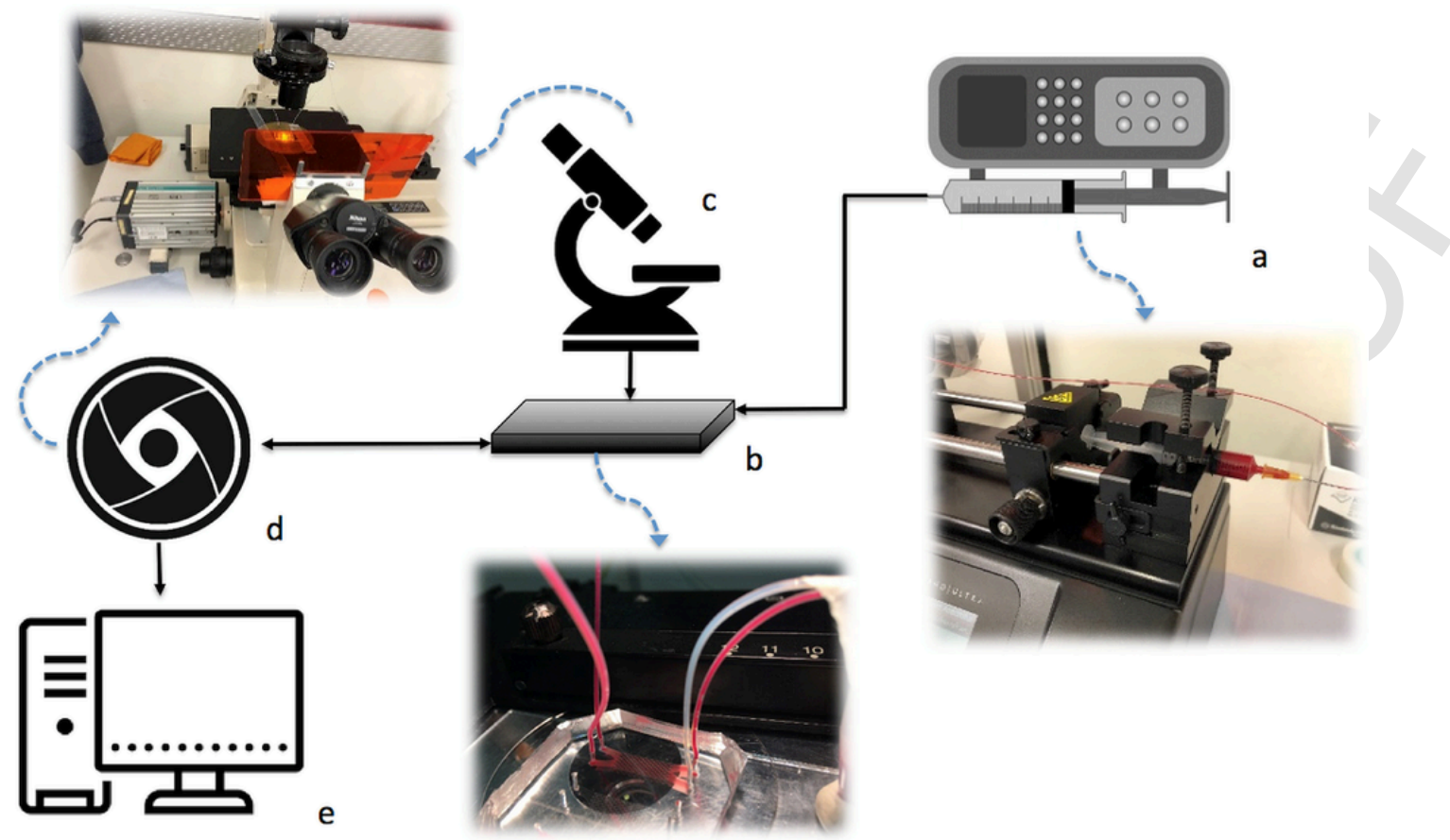

Fig. 3. Experimental setup. (a) Syringe pump loaded with blood sample; (b) microchannel perfusion; (c) microscope; (d) high-speed camera; (e) PC for postprocessing.

Ht was adjusted to different levels, ranging from 2 to $45 \%$. This range was selected to cover the typical haematocrit values in oxygenators which vary between 18 and $37 \%$, since various haemodilution steps can be used during cardiopulmonary bypass [18]. The Ht range was extended to lower values in our experiments for completeness. To minimize the sedimentation of RBCs before entering the microchannel, the syringe was turned upside down and the connection tubes tapped every two minutes.

\subsection{Image processing}

In order to quantify the RBC distributions observed in the microfluidic oxygenator models, a method similar to that reported in Sherwood et al. [24] was employed. 100 instantaneous RBC images were acquired with the high-speed camera at a sampling rate of $1 \mathrm{kHz}$ and were time averaged. The time averaged image intensity was normalized with the mean image intensity of the channel perfused at $0 \%$ $\mathrm{Ht}$, i.e. the maximum intensity corresponding to RBC free regions: $I_{N}=I_{a v} / I_{0 \% H t}$. This way, as higher Ht generates darker images (and hence lower $I_{a v}$ ), $\mathrm{I}_{\mathrm{N}} \leq 1$, with $\mathrm{I}_{\mathrm{N}}=1$ for $0 \% \mathrm{Ht}$. In order to make the intensity values more intuitive, so that regions of high haematocrit correspond to regions of high intensity the following normalized intensity was used: $I^{*}=1-I_{N}$. Thus, $I^{*}=0$ represents $0 \% \mathrm{Ht}$, increasing for higher concentrations. The image processing was performed with ImageJ [19] and Matlab (Mathworks). Fig. 4 illustrates the normalization process performed in a high aspect ratio straight microchannel with a cross sectional area of $40 \times 200 \mu \mathrm{m}$. Due to the aspect ratio, the $\mathrm{Ht}$ profile across the channel (in the plane of imaging) is almost flat resulting in a uniform $I^{*}$ distribution.

The sampling rate did not have a significant effect on the image intensity results, in contrast to the exposure time, i.e. the amount of time the camera lens is open to acquire the image, which has important effects in relation to the fluid velocity. In order to avoid blurred images that could compromise the $\mathrm{Ht}$ measurement, a constant exposure time of $50 \mu \mathrm{s}$ was used for all experiments.

\section{Results}

\subsection{Preliminary results}

Preliminary experiments using bovine blood revealed a heterogeneous RBC distribution at different scales of the channels. Starting at
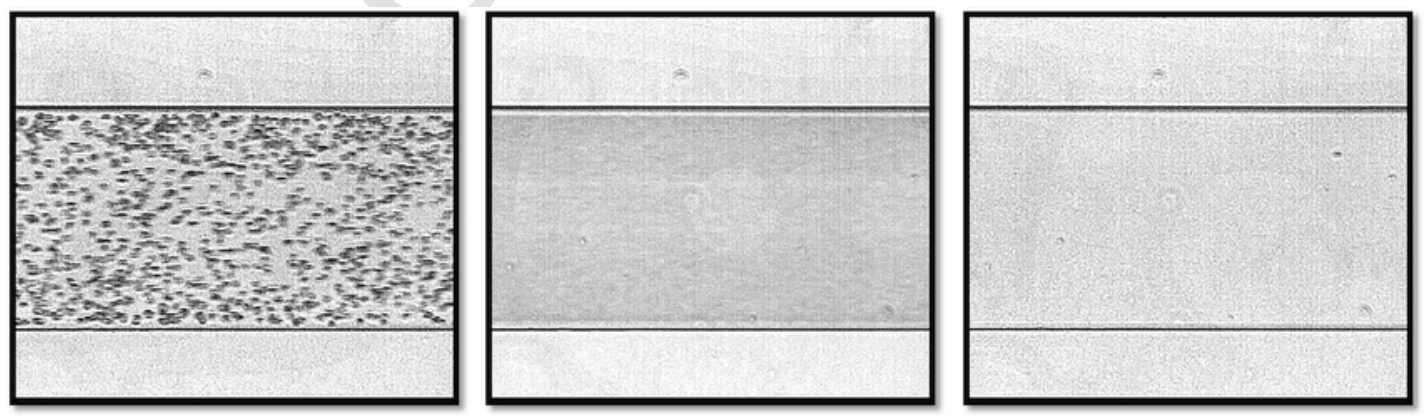

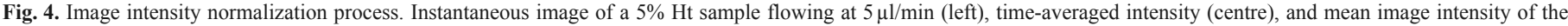
channel perfused with PBS only (right). 
the fibre level, images of RBC flows in various sections of channel c1 and at various haematocrits clearly show non-uniform RBC distributions, as indicated by the image intensity distributions in Fig. 5. The selected images illustrate sharp RBC concentration gradients between the fibres due to the flow field generated by the pin arrays. Regions depleted of RBCs can be seen in the fibre wakes and near the walls as expected.

Imaging of the larger scale microchannel, channel c2, shows how the non-uniform $\mathrm{RBC}$ concentration patterns propagate through the entire domain (Fig. 6) at both low and high haematocrits. The turn at the inlet of the array can further contribute to the heterogeneity in the fibre array and this can be illustrated in Fig. 6, where the main stream of the fluid hardly reaches the channel corner, thus causing RBC slowing down and sedimenting. In order to mimic specific conditions that can occur during normal functioning of the device (e.g. any disturbance in the homogeneity of the blood flow feeding the oxygenator), a non-uniform $\mathrm{Ht}$ distribution at the channel inlet was induced by purposely letting the RBC sediment in the syringe. This effect results in a complete separation of the two phases (RBCs and PBS) at certain locations of the channel, thus demonstrating that even the slightest inhomogeneity in local Ht will propagate in the whole microdevice (Fig. 7). Some asymmetries in RBC distributions observed in symmetric geometries (e.g. c1 and c3), most likely due to bistable nature of the flow in the array, provide additional testament of this phenomenon.

Finally, results in channel c3 (Fig. 8) also illustrate phase separation because of the flow field around the fibres and near the walls. The regions near the channel wall are not reached by RBCs, but only

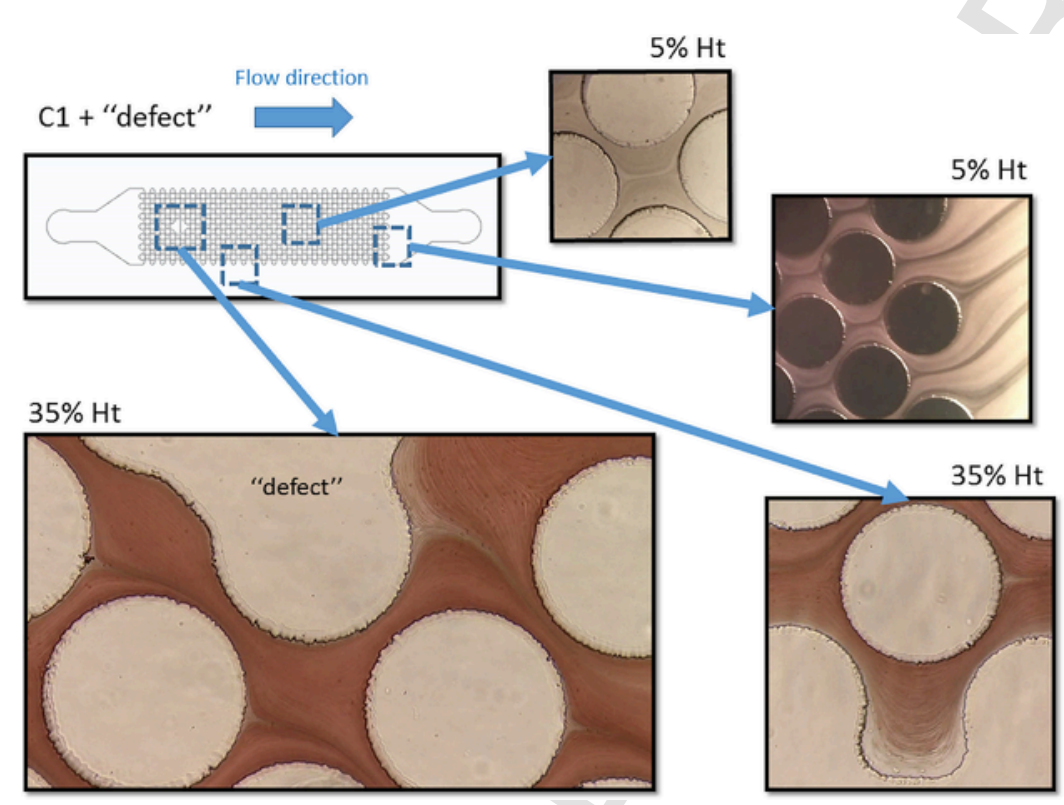

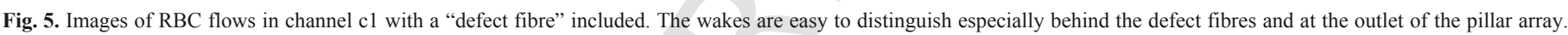

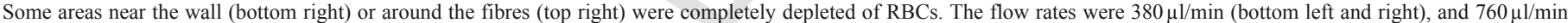
(top centre and right), corresponding to average velocities of 0.07 and $0.14 \mathrm{~m} / \mathrm{s}$ around the fibres.

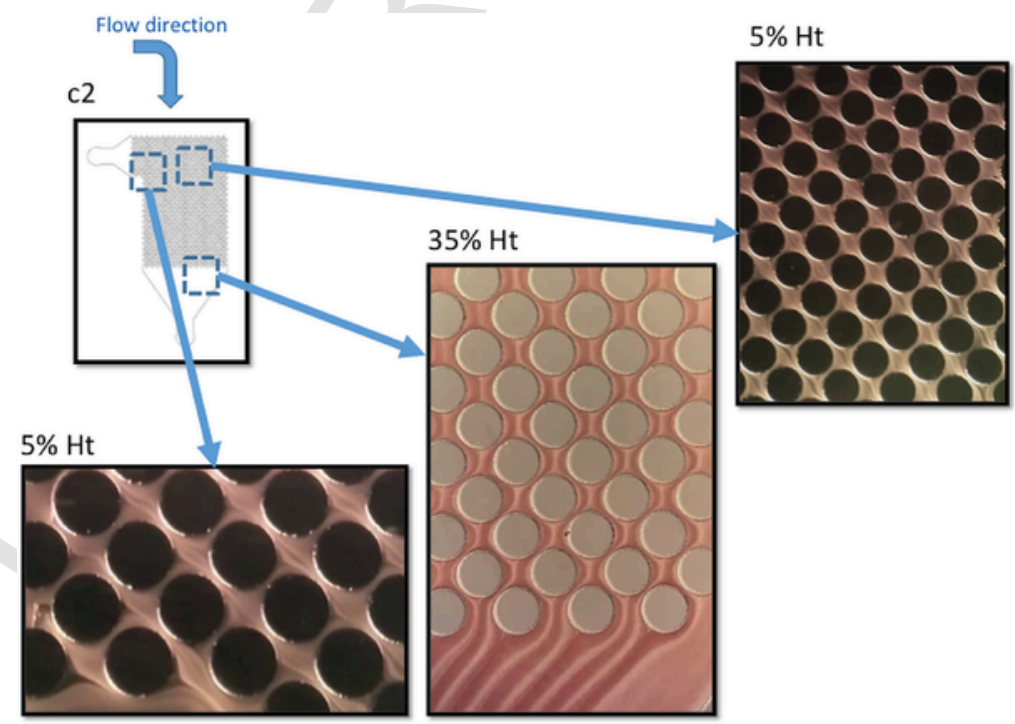

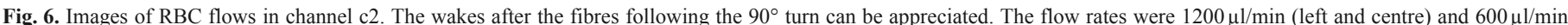
(right), corresponding to average velocities of 0.14 and $0.7 \mathrm{~m} / \mathrm{s}$ around the fibres. 


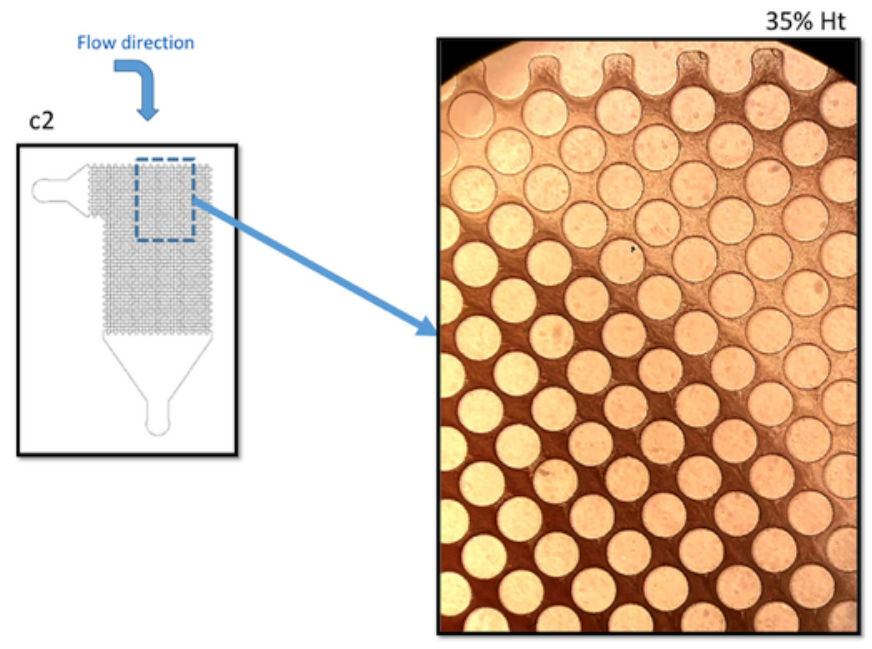

Fig. 7. Images of RBCs flows in channel c2, illustrating the impact of a non-uniform $\mathrm{RBC}$ distribution at the inlet. Inlet non-uniformities propagate across the channel, creating large regions in the array depleted of cells. The flow rate was $1000 \mu 1 / \mathrm{min}$ corresponding to an average velocity of $0.12 \mathrm{~m} / \mathrm{s}$ around the fibres.
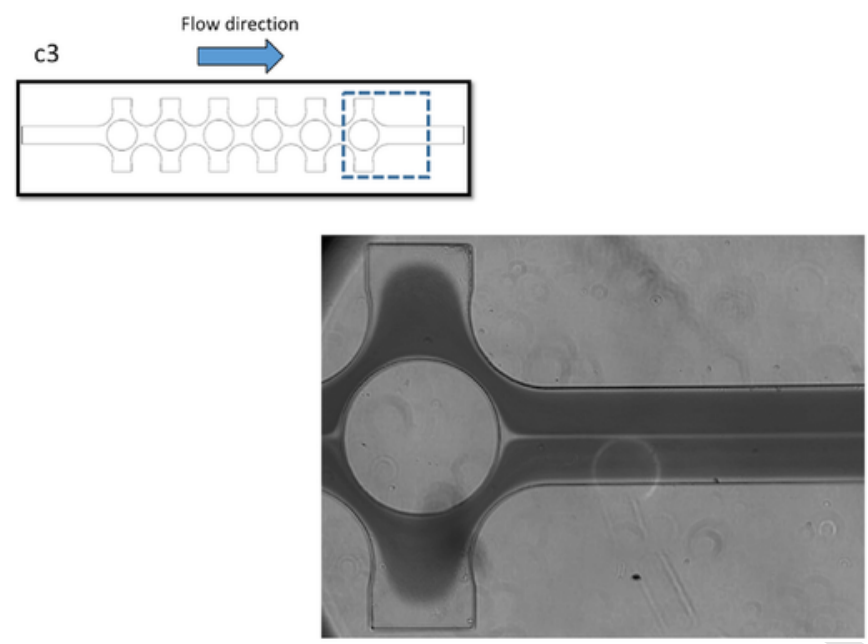

Fig. 8. Time averaged image of $\mathrm{RBC}$ flow in channel $\mathrm{c} 3$, showing a complete separation of phases near the walls, a clear non-homogeneous RBC distribution in the fibre contour, cell-depletion layers adjacent to the walls and a stabilized wake downstream of the fibre. The flow rate was $100 \mu \mathrm{l} / \mathrm{min}$, corresponding to an average velocity of $0.2 \mathrm{~m} / \mathrm{s}$ around the fibres.

by PBS. Similarly, RBC depleted regions are also observed in the boundary layers around the fibres, and along the wake centreline of the fibres resulting in a heterogeneous Ht distribution in the array. It is interesting to note that the phase separation in the wake became fully developed after the first obstacle row, due to the low Re, and persisted for a few fibre diameters downstream at the channel outlet.

\subsection{Quantitative analysis of image intensity}

The normalized intensity $I^{*}$ distribution was analyzed in different sections and for different flow conditions (velocities, feed Ht), including inter-fibre gaps, post-fibre wakes, or in gaps between fibres and walls. The results illustrate local haematocrit variations throughput the fibre arrays, with reduced $\mathrm{Ht}$ and almost cell depleted regions in the wake and near the walls. Fig. 9 shows a cell depletion region along the fibre wake centreline and near the wall of the fibres which is manifested by steep gradients in the intensity profiles across the gaps. Fig. 10 shows a linear haematocrit profile in the gap between fibres and the wall with steep gradients near the walls which are feed-haematocrit dependent and Fig. 11 shows haematocrit fluctuations across the outlet of the array as a result of the parallel wakes forming by the last rows of fibres.

\section{Discussion}

Motivated by the similar length scales in oxygenating bundles and the microvasculature, different microchannels were designed and manufactured as idealizations of complex 3D hollow fibre arrangements. These comprise staggered arrays of micropillars mimicking the fibres of the oxygenators. When the developed channels were perfused with different blood samples, heterogeneous distributions of RBCs were observed, with wake and near wall regions exhibiting a Ht close to zero. These results question the validity of the classic assumption of a homogeneous Ht inside blood oxygenators, which is consistently made when modeling these devices.

The observed differences in local haematocrit result from RBC dynamics and interactions in the flow field generated by the fibre array characterized by wakes and boundary layers near the walls. These fields generate a shear distribution within the micro pillar arrays, which affects the lateral migration of RBCs, enhancing the lift-induced effects [30,31]. These distributions imply that aggregation of $\mathrm{RBCs}$ is also likely despite the relatively high velocities in oxygenators, as the latter is both shear and haematocrit dependent. The observed local haematocrit distributions imply that blood oxygenation should vary throughout the blood path, considering the effects of RBC concentration on $\mathrm{O}_{2}$ diffusivity in blood [4,22]. Even though the cell depletion layers observed in these microfluidic arrays do not exactly reproduce those encountered in real oxygenating devices they provide some clues about the impact of such regions on oxygenator performance.

The small difference in the intensity values reported in Fig. 9 for the higher feed Ht seems to suggest that the impact of the cell-depleted regions on heterogeneity diminishes with Ht. Nevertheless, a complete understanding of haematocrit heterogeneity would require specific calibration curves to convert the recorded intensity maps in haematocrit values. Preliminary experiments, performed in a straight microchannel at varying feed $\mathrm{Ht}$, showed a non-linear dependency between RBC concentration and $I^{*}$ (Fig. 12), in agreement with previous works [20]. Calibration tests were performed using two different human blood samples perfused through a straight microchannel at velocities similar to those encountered in oxygenators. Calibration results show some variability between the two samples and a slight influence of flow velocity on $I^{*}$ which require further investigation. Nevertheless, the non-linear $I^{*}-H t$ relationship can still help to provide a preliminary interpretation of the results presented above. For example, Fig. 12 indicates that an increase of 0.1 in $I^{*}$ corresponds to an increase of about $5 \%$ in local $\mathrm{Ht}$ at low feed $\mathrm{Hts}$ (e.g. for feed $\mathrm{Ht}<15 \%$ ) but a threefold increase at higher feed Hts. Hence, the phase separation in the interstitial space between two fibres (e.g. at $\mathrm{L}^{*}=0.5$ ), that manifests as a sharp drop in I* values for a feed $\mathrm{Ht}$ of $15 \%$ (blue line in Fig. 9), also occurs at feed Ht of 35-45\% (purple line in Fig. 9). In the case reported in Fig. 9, for example, using the curve corresponding to fresh blood (Human-1 in Fig. 12) we would obtain a local Ht in the cell depleted region that is about 2-3 times lower than the remaining part of the RBC suspension.

As a result of the heterogeneity of $\mathrm{RBC}$ distribution, also the viscosity is expected to vary locally $[24,25]$, due to the complex rheology of blood that depends heavily on its particulate nature (i.e. haematocrit/microstructure), and hence the fluid dynamics in the fibre array are expected to differ from those predicted using the assumption of constant haematocrit throughout. Therefore, the particu- 


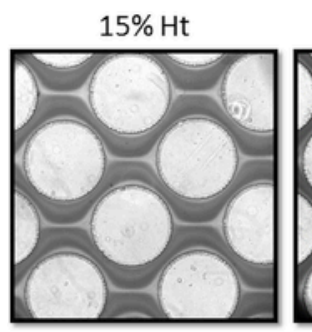

$25 \% \mathrm{Ht}$
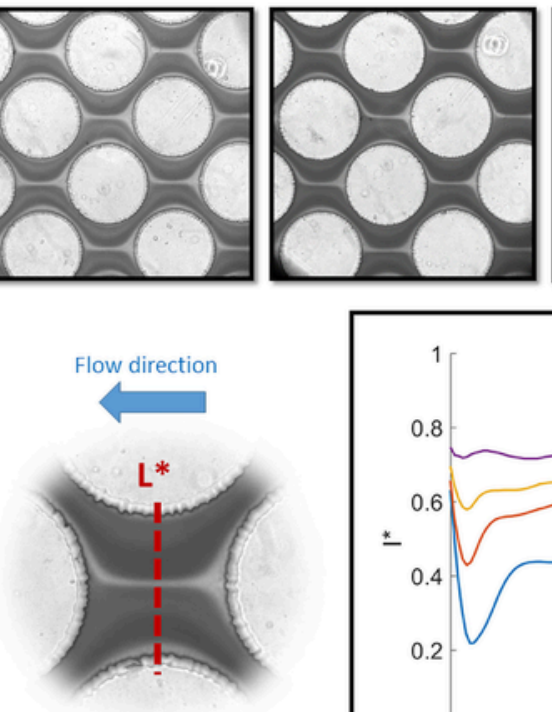

$35 \% \mathrm{Ht}$
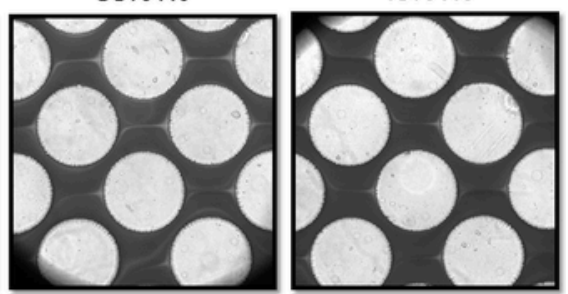

Intensity between two fibers

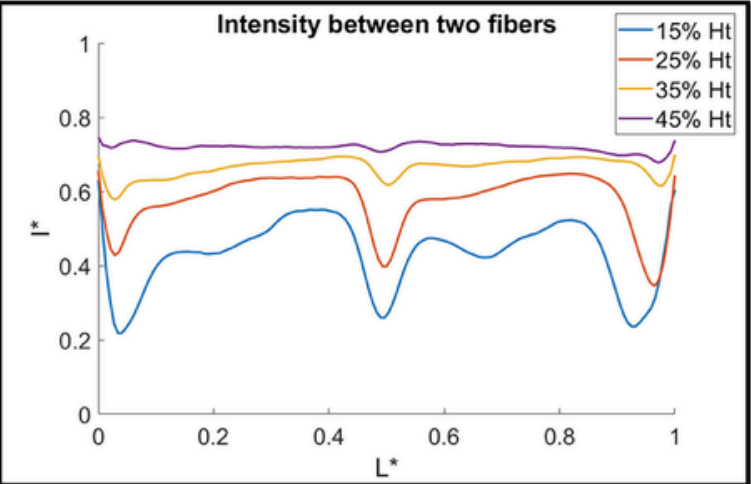

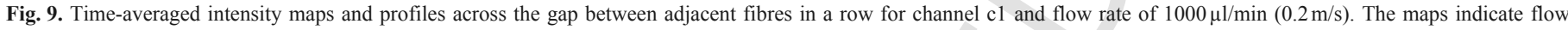

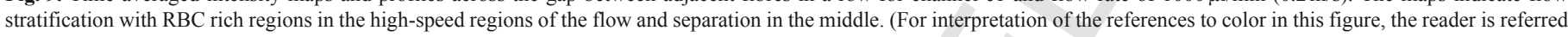
to the web version of this article.)
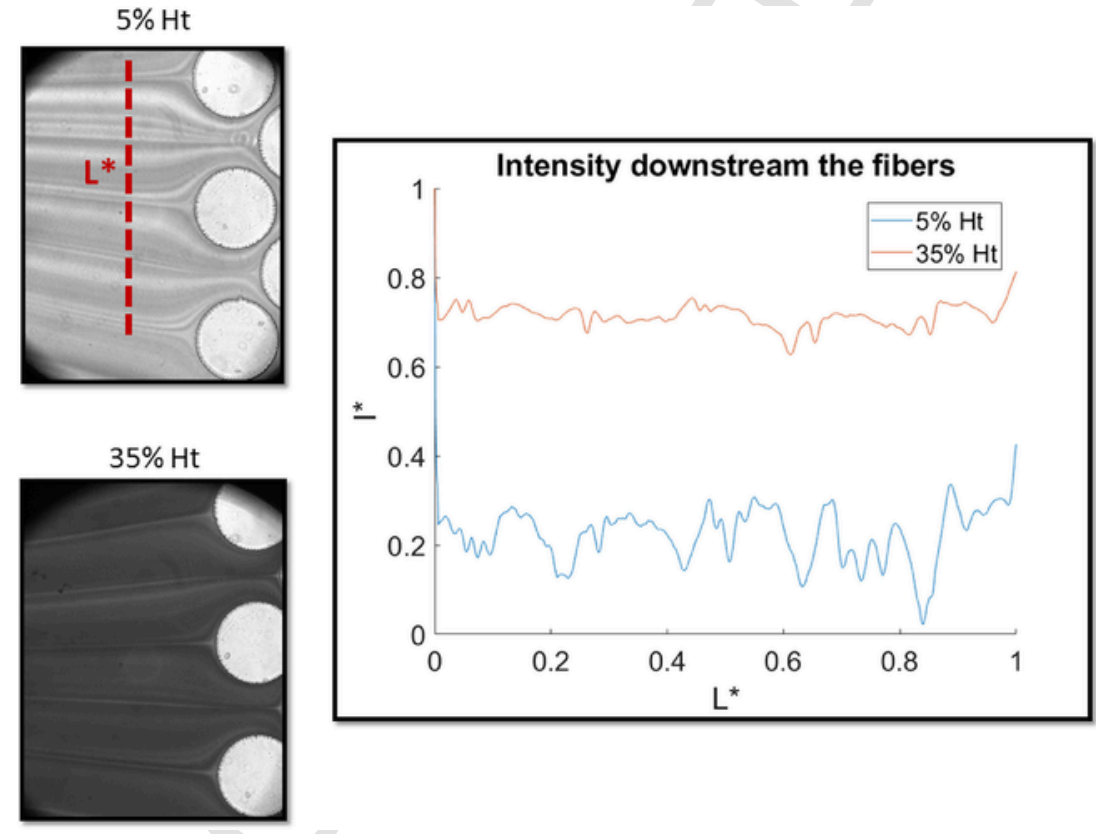

Fig. 10. Time-averaged intensity maps and profiles across the wakes formed downstream of the fibre array for channel $\mathrm{c} 1$ for a flow rate of $500 \mu 1 / \mathrm{min}(0.1 \mathrm{~m} / \mathrm{s})$.

late nature of blood should be considered for a more accurate modeling of the fluid dynamics and $\mathrm{O}_{2}$ transfer inside blood oxygenators.

All experiments on the same channel geometry were conducted with a RBC suspension from the same donor. However, some variability between different blood samples is likely to occur as blood composition and properties (e.g. deformability, aggregation) are patient specific. Tests using blood samples from transfusion bags, which had been stored for different amounts of time showed that image intensity values for equivalent feed $\mathrm{Ht}$ varied between the samples, as could be expected given the dependence of optical properties on factors like $\mathrm{O}_{2}$ saturation, temperature or osmolarity. In particular, sample noted as Human-1 corresponds to fresh blood whereas Human-2 to transfusion blood. Storage is known to alter several RBC properties, such as shape, membrane morphology and protein arrangement, metabolism, haemoglobin [27,28]. These alterations not only impact haemodynamics and physiology, but also affect the optical properties of cells [32].

Numerous variables related to the properties of blood, including inter-patient variations and types of clinical interventions, might affect the behavior of blood in these oxygenators. These effects are out 

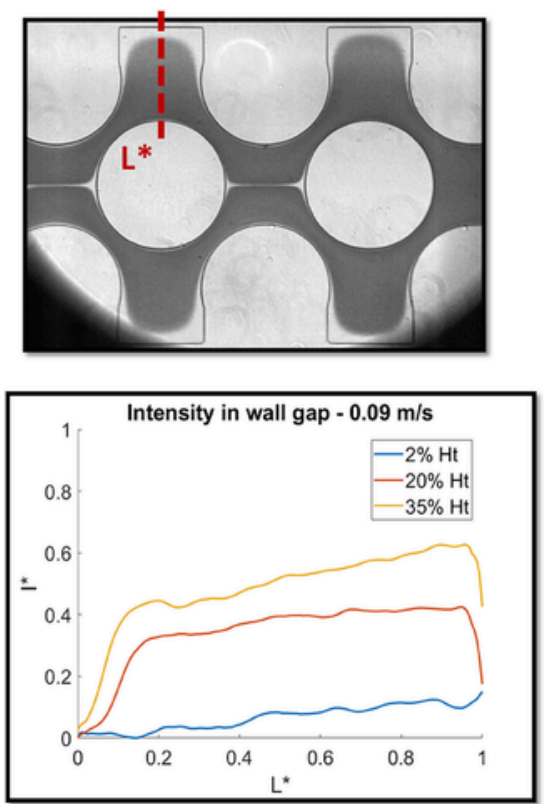

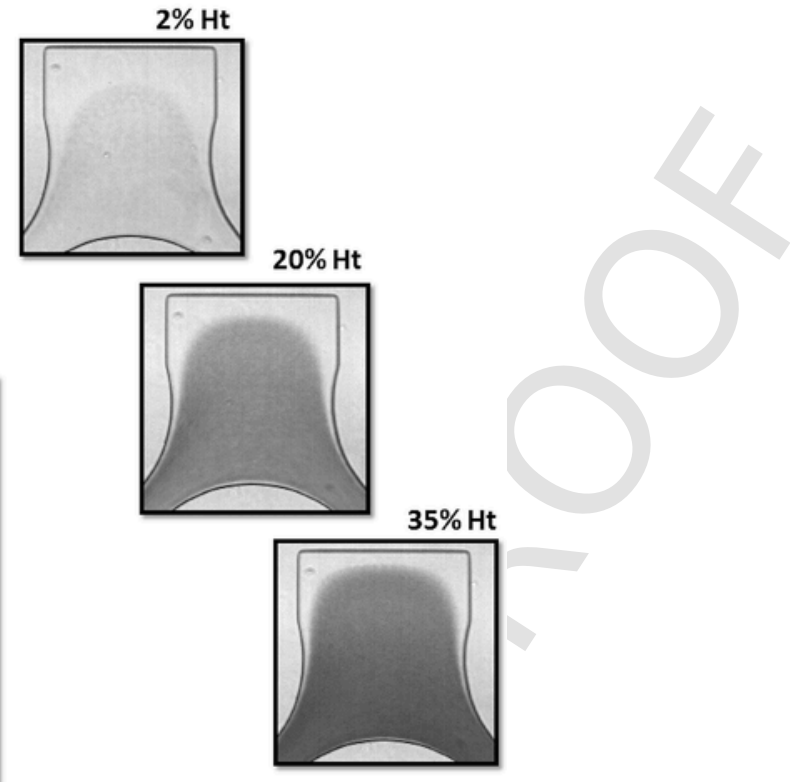

Fig. 11. Time-averaged intensity maps and profiles in the gap between a fibre and the channels wall for $\mathrm{c} 3$ for a flow rate of $70 \mu \mathrm{l} / \mathrm{min}(0.16 \mathrm{~m} / \mathrm{s})$.

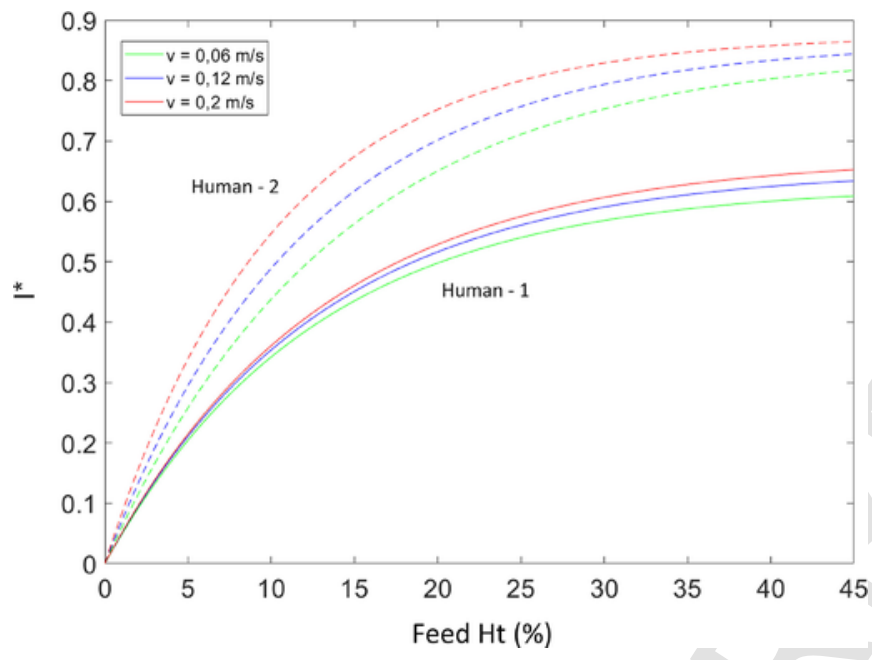

Fig. 12. Feed Ht-I* relationship of human RBC samples perfused at various flow velocities through a high aspect ratio straight microchannel with a cross sectional area of $40 \times 200 \mu \mathrm{m}$.

of the scope of this study, whose aim was to provide a proof-of-concept about the Ht heterogeneity occurring in oxygenator flow conditions and suggest the need to account for this during the oxygenator design phase. It is likely that such effects are present in current devices, but their net impact on the overall performance might be masked by other factors.

Finally, it should be noted that the present study was carried out under steady-state flow conditions and no attempt was made to introduce a pulsating flow mimicking the circulation. Use of a pulsatile flow would introduce the effect of frequency (Strouhal number) [29], thus requiring a redesign of the study. The flow inside the array is expected to be steady, as the corresponding Reynolds numbers in the study are not sufficient to induce vortex shedding in such confined geometries. Previous studies, performed at higher Re, indicate that effect of upstream flow pulsations might be limited to the first row in the array [33].

\section{Conclusion}

Even if the fluid dynamics of blood is largely studied in microcirculation, the effect of the particulate nature of blood during the design of oxygenating fibre bundles is rarely accounted for. In this work experimental evidence is presented that $\mathrm{RBC}$ distribution is not homogeneous in microdevices mimicking the dimensions and fluid dynamic characteristics of a fibre bundle. These phenomena are particularly evident between two consecutive fibres and near the channel wall. The presence of cell-depletion layers can affect the $\mathrm{O}_{2} / \mathrm{CO}_{2}$ mass transfer. A quantification of this effect can be possible by means of a CFD model that accounts for different diffusion coefficients in plasma and RBCs, thus looking for designs that take advantage of this phenomenon to increase the oxygenator performance.

Future work will involve refining the experimental data and use them to thoroughly validate a multiphase flow model for oxygenator design. Moreover, we should focus on alternative, more realistic three-dimensional fibre bundles and more sophisticated designs that will allow examining the effects of haematocrit heterogeneity and patient variability and their effects on gas transport in a controlled manner, tapping on and exploring the numerous possibilities of microfluidic technologies for artificial organs (in particular, lungs), described in previous studies [23].

\section{Declaration of Competing Interest}

The authors have no professional or financial conflicts of interest to disclose.

\section{Acknowledgments}

This research was funded by the European Commission through the H2020 Marie Skłodowska-Curie European Training Network H2020-MSCA-ITN-2014 VPH-CaSE, www.vph-case.eu, GA No. 642612.

Part of this work was performed at PoliFab, the micro- and nanofabrication facility of Politecnico di Milano (http://www.polifab. 
polimi.it/). The authors would like to thank Ms Alessia Romeo for her valuable technical support.

\section{Ethical approval}

The study was approved by the Southeast London Research Ethics Committee (ref: 10/H0804/21).

\section{References}

[1] S.R. Wickramasinghe, A.R. Goerke, J.D. Garcia, B. Han, Designing blood oxygenators, Ann N Y Acad Sci 984 (2003) 502-514.

[2] S.R. Wickramasinghe, M.J. Semmens, E.L. Cussler, Mass-transfer in various hollow fiber geometries, J Membr Sci 69 (1992) 235-250.

[3] K. Nagase, F. Kohori, K. Sakai, Oxygen transfer performance of a membrane oxygenator composed of crossed and parallel hollow fibers, Biochem Eng J 24 (2005) 105-113.

[4] M. Hormes, R. Borchardt, I. Mager, T. Schmitz-Rode, M. Behr, U. Steinseifer, A validated CFD model to predict $\mathrm{O} 2$ and $\mathrm{CO} 2$ transfer within hollow fiber membrane oxygenators, Int J Artif Organs 34 (3) (2011) 317-325.

[5] R. Graefe, R. Borchardt, J. Arens, P. Schlanstein, T. Schmitz-Rode, U. Steinseifer, Improving oxygenator performance using computational simulation and flow field-based parameters, Artif Organs 34 (2010) 930-936.

[6] K.W.Q. Low, R. van Loon, S.A. Rolland, J. Sienz, Pore-scale modeling of non-Newtonian shear-thinning fluids in blood oxygenator design, J Biomech Eng 138 (2016), 051001.

[7] J. Zhang, X. Chen, J. Ding, K.H. Fraser, M.E. Taskin, B.P. Griffith, Z.J. Wu, Computational study of the blood flow in three types of 3D hollow fiber membrane bundles, J Biomech Eng 135 (12) (2013), 121009.

[8] P.C. Sousa, F.T. Pinho, M.A. Alves, M.S.N. Oliveira, A review of hemorheology: measuring techniques and recent advances, Korea Aust Rheol J 28 (2016) $1-22$.

[9] T.W. Secomb, Blood flow in the microcirculation, Annu Rev Fluid Mech 49 (2017).

[10] F. Yilmaz, M.Y. Gundogdu, A critical review on blood flow in large arteries; relevance to blood rheology, viscosity models and physiologic conditions, Kore-Australia Rheol J 20 (2008) 197-211.

[11] A.R. Pries, T.W. Secomb, P. Gaehtgens, Biophysical aspects of blood flow in the microvasculature, Cardiovasc. Res. 32 (1996) 654-667.

[12] R. Fahraeus, The suspension stability of the blood, Physiol. Rev. 9 (1929) 241-274.

[13] R. Fahraeus, T. Lindqvist, The viscosity of the blood in narrow capillary tubes, Am J Physiol 96 (1931) 562-568.

[14] A. Kaesler, M. Rosen, T. Schmitz-Rode, U. Steinseifer, J. Arens, Computational modeling of oxygen transfer in artificial lungs, Artif Organs 42 (2018) 786-799.

[15] M. Lake, M. Lake, C. Narciso, K. Cowdrick, T. Storey, S. Zhang, D. Hoelzle, Microfluidic device design, fabrication, and testing protocols, Protoc Exch (2015).
[16] M.E. Taskin, K.H. Fraser, T. Zhang, B.P. Griffith, Z.J. Wu, Micro-scale modeling of flow and oxygen transfer in hollow-fiber membrane bundle, J. Memb. Sci. 362 (2010) 172-183.

[17] J.R. Zierenberg, H. Fujioka, K.E. Cook, J.B. Grotberg, Pulsatile flow and oxygen transport past cylindrical fiber arrays for an artificial lung: computational and experimental studies, J. Biomech. Eng. 130 (2008), 031019.

[18] R. Muraki, A. Hiraoka, K. Nagata, K. Nakajima, T. Oshita, M. Arimichi, G. Chikazawa, H. Yoshitaka, T. Sakaguchi, Novel method for estimating the total blood volume: the importance of adjustment using the ideal body weight and age for the accurate prediction of haemodilution during cardiopulmonary bypass, Interact Cardiovasc Thorac Surg. 27 (6) (2018) 802-807.

[19] C.A. Schneider, W.S. Rasband, K.W. Eliceiri, NIH image to ImageJ: 25 years of image analysis, Nat. Methods 9 (7) (2012) 671-675.

[20] J.M. Sherwood, D. Holmes, E. Kaliviotis, S. Balabani, Spatial distributions of red blood cells significantly alter local haemodynamics, PLoS ONE 9 (6) (2014), e100473.

[21] H. Stauber, D. Waisman, N. Korin, J. Sznitman, Red blood cell dynamics in biomimetic microfluidic networks of pulmonary alveolar capillaries, Biomicrofluidics 11 (2017), 014103.

[22] S.N. Vaslef, L.F. Mockros, R.W. Anderson, R.J. Leonard, Use of a mathaematical model to predict oxygen transfer rates in hollow fiber membrane oxygenators, Asaio J 40 (1994) 990-996.

[23] J. Potkay, The promise of microfluidic artificial lungs, Lab Chip 14 (2014) 4122

[24] J.M. Sherwood, E. Kaliviotis, J. Dusting, S. Balabani, Hematocrit, viscosity and velocity distributions of aggregating and non-aggregating blood in a bifurcating microchannel, Biomech Model Mechanobio 113 (2014) 259-273.

[25] E. Kaliviotis, J. Dusting, S. Balabani, Spatial variation of blood viscosity: modelling using shear fields measured by a $\mu$ PIV based technique, Med Eng Phys (2011) 33824-33831.

[26] L.G. Rigat-Brugarolas, A. Elizalde-Torrent, M. Bernabeu, M. De Niz, L. Martin-Jaular, C. Fernandez-Becerra, A. Homs-Corbera, J. Samitier, H.A. del Portillo, A functional microengineered model of the human spleen on-on-a-chip, Lab Chip 14 (2014) 1715

[27] D. Orlov, K. Karkouti, The pathophysiology and consequences of red blood cell storage, Anaesthesia 70 (Suppl. 1) (2015) 29-37.

[28] A. D'Alessandro, G. Liumbruno, G. Grazzini, L. Zolla, Red blood cell storage: the story so far, Blood Transfus 8 (2010) 82-88.

[29] L. Cortelezzi, S. Ferrari, G. Dubini, A scalable active micro-mixer for biomedical applications, Microfluid Nanofluid 21 (31) (2017).

[30] T.M. Geislinger, T. Franke, Hydrodynamic lift of vesicles and red blood cells inflow-from Fåhræus and Lindqvist to microfluidic cell sorting, Adv Colloid Interf Sci 208 (2014) 161-176.

[31] R. Lima, T. Ishikawa, Y. Imai, T. Yamaguchi, Blood flow behavior in microchannels: past, current and future trends, Single and two-phase flows on chemical and biomedical engineering, Bentham Science, 2012513-547.

[32] N. Bosschaart, G.J. Edelman, M.C.G. Aalders, T.G. van Leeuwen, D.J. Faber, A literature review and novel theoretical approach on the optical properties of whole blood, Lasers Med Sci 29 (2) (2014) 453-479.

[33] E. Konstantinidis, D. Castiglia, S. Balabani, M. Yianneskis, On the flow and vortex shedding characteristics of an in-line tube bundle in steady and pulsating crossflow, Chem Eng Res Des 78 (8) (2000) 1129-1138. 